\title{
The local instability of steady astrophysical flows with non circular streamlines with application to differentially rotating disks with free eccentricity
}

\author{
J. C. B. Papaloizou
}

\author{
Astronomy Unit, Queen Mary, University of London, Mile End Rd, London E1 4NS, UK \\ e-mail: jcbp@maths.qmw.ac.uk
}

Received 3 September 2004 / Accepted 22 November 2004

\begin{abstract}
We carry out a general study of the stability of astrophysical flows that appear steady in a uniformly rotating frame. Such a flow might correspond to a stellar pulsation mode or an accretion disk with a free global distortion giving it finite eccentricity.

We consider perturbations arbitrarily localized in the neighbourhood of unperturbed fluid streamlines. When conditions do not vary around them, perturbations take the form of oscillatory inertial or gravity modes. However, when conditions do vary so that a circulating fluid element is subject to periodic variations, parametric instability may occur. For nearly circular streamlines, the dense spectra associated with inertial or gravity modes ensure that resonance conditions can always be satisfied when twice the period of circulation round a streamline falls within.

We apply our formalism to a differentially rotating disk for which the streamlines are Keplerian ellipses, with free eccentricity up to 0.7 , which do not precess in an inertial frame. We show that for small $e$, the instability involves parametric excitation of two modes with azimuthal mode number differing by unity in magnitude which have a period of twice the period of variation as viewed from a circulating unperturbed fluid element. Instability persists over a widening range of wave numbers with increasing growth rates for larger eccentricities.

The nonlinear outcome is studied in a follow up paper which indicates development of small scale subsonic turbulence.
\end{abstract}

Key words. accretion, accretion disks - instabilities - hydrodynamics - celestial mechanics - planets and satellites: formation stars: oscillations

\section{Introduction}

In Astrophysics one is often concerned with the stability of a system undergoing some regular global steady motion. Possible examples are stars undergoing a pulsation that may be either freely excited or forced through tidal interaction in a binary system. Other examples, which are more the focus of this paper, are differentially rotating disks which have been either set up or perturbed into a state in which the orbital motion about the centre is non circular. Systems of this type are known to be susceptible to parametric instabilities which involve a resonance between the periods of normal modes of oscillation and a period associated with the unperturbed motion. For examples of studies related to stellar oscillations see Vandakurov (1967), Papaloizou (1973), Papaloizou \& Pringle (1980), Kumar \& Goodman (1996), and Wu \& Goldreich (2001).

The presence of accretion disks in which the orbits are eccentric have been suggested in a number of contexts. The production of such disks by the tidal disruption of stars orbiting around AGN has been considered by Gurzadyan \& Ozernoy (1979). A model to explain the long term spectral variability of Be stars as being due to global one-armed $(m=1)$ oscillations of equatorial disks has been developed (e.g. Okazaki 1991; Papaloizou et al. 1992). Eccentric disks have also been proposed as an explanation for the superhump phenomenon in SU UMA stars (Whitehurst 1988).

A general theory to describe the slow viscous evolution of disks with modest to large eccentricities has been developed by Ogilvie (2001). This confirms the possibility of long lived structures of this type within that context.

The stability of the non circular motions induced in an accretion disk by a binary companion has been studied in pioneering work by Goodman (1993) and Ryu \& Goodman (1994). These authors studied the fast $m=2$ forced distortions that propagate around with the companion. A parametric instability was found that lead to turbulence in the context of local two dimensional modelling in a shearing box.

In this paper we are concerned with extending studies of the stability of disks with non circular motions to more general situations such as global disks with free global $m=1$ modes which are almost stationary in an inertial frame.

The recent discovery of a number of extrasolar giant planets orbiting around nearby solar-type stars (Mayor \& Queloz 1995; 
Marcy \& Butler 1998, 2000) with high orbital eccentricities in the range $0.0 \lesssim e \lesssim 0.93$ has led to the consideration of the proposal that a giant planet orbiting in and interacting with a protostellar disk (e.g. Goldreich \& Tremaine 1980; Ward 1997) could undergo an instability that would cause its eccentricity to increase. (Artymowicz 1992; Lin \& Papaloizou 1993; Papaloizou et al. 2001; Goldreich \& Sari 2003). However, the free modes in such an interaction are likely to be coupled to the disk which participates with significant eccentricity in a global $m=1$ mode. (Papaloizou 2002; Goldreich \& Sari 2003). Accordingly the behaviour and in particular the damping of free eccentricity in differentially rotating disks becomes an issue. It is one of the purposes of this and a follow up paper (Papaloizou 2005) to study the instabilities of free global disk normal modes corresponding to free eccentricity and their non linear outcome. Although there may be major regions of protostellar disks that support magnetic fields leading to turbulence through the Magnetorotational instability (Balbus \& Hawley 1991), because of dependence on the existence of external sources of ionization and complex chemistry, the extent of such regions is highly uncertain (e.g. Gammie 1996; Fromang et al. 2002). Accordingly for this first study we have considered disks with eccentricity in the hydrodynamic limit. This is of general theoretical interest because even in that limit there are potential local instabilities of disks with non circular but regular streamlines that could lead to turbulence and enhanced dissipation (Goodman 1993). Although uncertain, it is likely that the presence of magnetic fields would enhance any decay rate found in the hydrodynamic limit. However, this will be studied in a separate investigation.

In this paper we develop a general formalism for the study of the local stability of astrophysical flows possibly in stars or disks that appear steady in a uniformly rotating frame.

We adopt a local Lagrangian approach and consider perturbations localized near unperturbed fluid streamlines. These are shown to be governed by a pair of second order ordinary differential equations that give the evolution of the localized disturbance in the neighbourhood of a fluid element as a function of time. For streamlines on which conditions vary as a fluid element circulates, the coefficients are periodic functions of time. The parametric excitation of inertial or gravity modes readily occurs.

We solve these equations and delineate the instability for an example for which the streamlines are Keplerian ellipses, with free eccentricity up to 0.7 .

The nonlinear outcome of such instabilities in disks supporting global modes which endow them with eccentricity is studied in a follow up paper which indicates development of small scale subsonic turbulence.

The plan of the paper is as follows: In Sect. 2 we give the Basic equations. In Sect. 3 we describe the steady state flow conditions of the systems whose stability we analyse, in the context of a model with streamlines that are Keplerian ellipses. In Sect. 4 we discuss the general linear stability analysis and go on to describe the solution procedure used in the local limit in general terms relating the spectra found in the local problem to that of the global one in Appendix C. In Sect. 5 this is applied to the case when there are circular streamlines in the unperturbed steady state. In Sect. 6 this is extended to a steady state model with streamlines taking the form of Keplerian ellipses. Numerical and analytic solution of the governing equations are described in Sect. 7 for the Keplerian streamline case while emphasizing the general nature of the behaviour. Finally in Sect. 8 we discuss our results

\section{Basic equations}

We work in a rotating frame which rotates with angular velocity $\boldsymbol{\Omega}_{P}$ relative to an inertial frame.

We adopt a system of cylindrical coordinates $(r, \varphi, z)$ for which the origin is at the centre of mass and the angular velocity is directed along the $z$ axis such that $\boldsymbol{\Omega}_{\boldsymbol{P}}=\left(0,0, \Omega_{P}\right)$.

In the absence of magnetic fields, the equation of motion may be written in the form

$\frac{\partial \boldsymbol{v}}{\partial t}+\boldsymbol{v} \cdot \nabla \boldsymbol{v}+2 \boldsymbol{\Omega}_{\boldsymbol{P}} \times \boldsymbol{v}+\boldsymbol{\Omega}_{\boldsymbol{P}} \times\left(\boldsymbol{\Omega}_{\boldsymbol{P}} \times \boldsymbol{r}\right)=\boldsymbol{F}=-\frac{\nabla P}{\rho}-\nabla \Psi$.

Here the velocity is $\boldsymbol{v}=\left(v_{r}, v_{\varphi}, v_{z}\right)$. The left hand side includes contributions from the acceleration of a fluid element and coriolis and centrifugal accelerations arising because of the rotating frame. The acceleration of a fluid element may be written using the convective derivative taken moving with the fluid in the form

$\frac{D v}{D t}=\frac{\partial v}{\partial t}+\boldsymbol{v} \cdot \nabla v$

The system is subject to a force per unit mass $\boldsymbol{F}$ which has components due to pressure, $P$, and gravity, with the gravitational potential being $\Psi$. The density is $\rho$.

In addition we have the continuity equation

$\frac{\partial \rho}{\partial t}+\nabla \cdot(\rho \boldsymbol{v})=0$

\section{Steady state configurations}

We here consider the stability of steady state configurations for which $\partial / \partial t \equiv 0$. For these Eq. (1) gives

$\boldsymbol{v} \cdot \nabla \boldsymbol{v}+2 \mathbf{\Omega}_{\boldsymbol{P}} \times \boldsymbol{v}+\mathbf{\Omega}_{\boldsymbol{P}} \times\left(\boldsymbol{\Omega}_{\boldsymbol{P}} \times \boldsymbol{r}\right)=\boldsymbol{F}=-\frac{\nabla P}{\rho}-\nabla \Psi$.

In general this allows for a steady state flow $\boldsymbol{v}$ as viewed in a rotating frame.

A very simple possible motion is non uniform rotation for which $\boldsymbol{v}=\left(0, v_{\varphi}(r), 0\right)$ corresponding to circular motion about the $z$ axis at a rate which is a function of $r$.

Other examples could correspond to a normal mode of stellar oscillation as viewed in a frame rotating with the modal pattern speed or an accretion disk with a global eccentricity.

\subsection{Cylindrical disks}

In the context of differentially rotating accretion disks we consider fluid circulating in Keplerian ellipses in a cylindrical potential. For this $\boldsymbol{\Omega}_{\boldsymbol{P}}=0$ and $\Psi=-G M_{*} / r$. Here $M_{*}$ is a central 
mass and $G$ the gravitational constant. Disk self gravity is neglected. If in addition the pressure, $P$, is constant, we have

$\boldsymbol{v} \cdot \nabla \boldsymbol{v}=-\nabla \Psi$.

This model can approximate conditions near the midplane of a vertically stratified disk if $\partial v_{z} / \partial z=0$ there and with a slight modification if it is not.

Equation (5) applies to particle motion under only the potential $\Psi$. Thus a solution can be found from the Keplerian trajectory

$\frac{a\left(1-e^{2}\right)}{r}=1+e \cos \varphi$,

where the semi major axis $a$ and the eccentricity $e$ are constant on a streamline being a particle trajectory. On such a trajectory the velocity components are given by

$v_{r}=e \sin \varphi \sqrt{\frac{G M_{*}}{r(1+e \cos \varphi)}}$,

and

$v_{\varphi}=\sqrt{\frac{G M_{*}(1+e \cos \varphi)}{r}}$.

The eccentricity is constant on a stream line and may be specified as an arbitrary function of $a$. Equation (6) may then be solved for $e$ as a function of $r$ and $\varphi$. The density may then be found from the steady state continuity equation $\nabla \cdot(\rho v)=0$. In the special case when $e$ is constant, the continuity equation is satisfied for $\rho$ being a general function of its arguments of the form

$\rho=\rho(r(1+e \cos \varphi), z)$.

For the work presented later on we adopt a locally isothermal equation of state such that

$P=\rho c_{\mathrm{s}}^{2}(r)$.

The sound speed $c_{\mathrm{s}}(r)$ is taken to be a fixed function of $r$. As above we adopt a cylindrical disk model for which vertical stratification is neglected (e.g. Hawley 2000; Steinacker \& Papaloizou 2002) and the potential is independent of $z$ such that $\Phi=-G M_{*} / r$. We note again that this model can describe conditions close to the midplane of a stratified disk which is all that is required in order to perform a local stability analysis of the type we consider here. When $\rho$ is constant and $c_{\mathrm{s}}^{2}(r) \propto 1 / r$, Keplerian ellipses are possible streamlines that form a stationary pattern in the inertial frame.

\subsection{Properties of streamlines}

We study the stability of the steady state configurations described above, below. We focus on perturbations that are localized on particular streamlines and we assume that the configuration of steady state streamlines is smooth enough that at least locally the fluid volume can be filled with stream tubes that can be used to define a volume element $\mathrm{d} V=\mathrm{d} \mathcal{A d} s$, with $\mathrm{d} \mathcal{A}$ being the stream tube area element normal to a streamline with element of length $\mathrm{d} s$. Then the steady flow condition $\nabla \cdot \rho \boldsymbol{v}=0$ on a streamline may be written $\mathrm{d}(\rho|\boldsymbol{v}| \mathcal{A}) / \mathrm{d} s=0$. We also suppose that two independent invariants can be defined on each streamline. For such invariants $\zeta_{i}, i=1,2$

$\boldsymbol{v} \cdot \nabla \zeta_{i}=0$

In the simple case of differential rotation about the $z$ axis, the stream lines are circles centred on the $z$ axis. The invariants can be taken to be $r$ and $z$, the radius of a particular circle and its height $z$ respectively. Similarly for the cylindrical potential models with streamlines being Keplerian ellipses. The invariants could be taken to be $z$ along with the semi-major axis or eccentricity, both of which are constant on a streamline.

\section{Linear stability analysis}

\subsection{General formalism}

We now study the stability of steady state flows of the type introduced above. The most convenient formalism is the Lagrangian approach developed by Lynden-Bell \& Ostriker (1967). Following these authors we introduce the Lagrangian variation $\Delta$ such that any state variable $Q$ is perturbed following a fluid element from its value appropriate to the steady state such that

$Q \rightarrow Q+\Delta Q$

The Lagrangian displacement is introduced as the Lagrangian variation of the position vector of a fluid element so that

$\Delta \boldsymbol{r}=\boldsymbol{\xi}$.

The Lagrangian variation of the velocity is then

$\Delta v=\frac{D \xi}{D t}$,

where the convective derivative of $\boldsymbol{\xi}$ is

$\frac{D \xi}{D t}=\frac{\partial \xi}{\partial t}+\boldsymbol{v} \cdot \nabla \boldsymbol{\xi}$,

and as shown by Lynden-Bell \& Ostriker (1967)

$\Delta\left(\frac{D v}{D t}\right)=\frac{D \Delta v}{D t}$.

The Eulerian variation, $Q^{\prime}$ in $Q$ seen in a fixed coordinate system resulting from $\Delta Q$ is given by

$Q^{\prime}=\Delta Q-\xi \cdot \nabla Q$.

To obtain the basic governing equations for the linear stability problem, we take the Lagrangian variation of the equation of motion (1) which gives.

$\frac{D^{2} \boldsymbol{\xi}}{D t^{2}}+2 \boldsymbol{\Omega}_{\boldsymbol{P}} \times \frac{D \boldsymbol{\xi}}{D t}+\boldsymbol{\Omega}_{\boldsymbol{P}} \times\left(\boldsymbol{\Omega}_{\boldsymbol{P}} \times \boldsymbol{\xi}\right)=\Delta \boldsymbol{F}$.

Using Eq. (17) we obtain

$\Delta \boldsymbol{F}=-\frac{\nabla P^{\prime}}{\rho}+\frac{\rho^{\prime}}{\rho^{2}} \nabla P-\boldsymbol{\xi} \cdot \nabla\left(\frac{\nabla P}{\rho}+\nabla \Psi\right)$. 
We comment that because of the local nature of the perturbations considered, we use the Cowling approximation in which variation of the gravitational potential is neglected.

The Lagrangian variation in the density is related to the Lagrangian displacement through

$\Delta \rho=\rho^{\prime}+\boldsymbol{\xi} \cdot \nabla \rho=-\rho \nabla \cdot \boldsymbol{\xi}$

The pressure and density variations could be related by the adiabatic condition

$\frac{\Delta P}{P}=\frac{\Gamma \Delta \rho}{\rho}$,

where $\Gamma$ is the first adiabatic exponent. For simplicity we shall assume this to be constant below, however this is not an essential requirement for what follows.

One can seek solutions for which the time dependence is through a factor $\exp (i \sigma t)$ where $\sigma$ is the eigenfrequency associated with a normal mode and after taking all time derivatives divide this factor out. Then

$\frac{D \boldsymbol{\xi}}{D t} \equiv i \sigma \boldsymbol{\xi}+\boldsymbol{v} \cdot \nabla \boldsymbol{\xi}$

and

$\frac{D^{2} \boldsymbol{\xi}}{D t^{2}} \equiv\left(-\sigma^{2}+2 i \sigma \boldsymbol{v} \cdot \nabla \boldsymbol{\xi}+\boldsymbol{v} \cdot \nabla(\boldsymbol{v} \cdot \nabla \boldsymbol{\xi})\right)$.

Adopting Eqs. (22)-(23), the linear stability problem (18) can be reduced to the operator equation (see Lynden-Bell \& Ostriker 1967)

$\boldsymbol{L}(\boldsymbol{\xi})=-\sigma^{2} \boldsymbol{\xi}+\sigma \boldsymbol{B}(\boldsymbol{\xi})+\boldsymbol{C}(\boldsymbol{\xi})=0$,

where, if the adiabatic condition (21) is used, the operators $\boldsymbol{B}, \boldsymbol{C}$ are self adjoint with weight $\rho$. That is for two arbitrary displacements $(\boldsymbol{\xi}, \boldsymbol{\eta})$ and standard boundary conditions

$(\boldsymbol{\eta}, \boldsymbol{C}(\boldsymbol{\xi})) \equiv \int_{V} \rho \boldsymbol{\eta}^{*} \cdot \boldsymbol{C}(\boldsymbol{\xi}) \mathrm{d} V=\int_{V} \rho \boldsymbol{\xi} \cdot \boldsymbol{C}^{*}\left(\boldsymbol{\eta}^{*}\right) \mathrm{d} V$

with the integration being taken over the domain of the fluid and a similar relation holding for $\boldsymbol{B}$. This also defines the inner product when $\boldsymbol{C}=\boldsymbol{I}$, the identity.

This self adjoint property, although useful for some purposes is not essential to the analysis carried out in this paper which can in fact be extended to equations of state for which it does not hold (see below). Accordingly we shall not consider the self-adjoint formalism further here.

\subsection{Local analysis}

We here consider perturbations that are localized on streamlines. To do this we suppose that any perturbation quantity is of the form

$\Delta Q=(\Delta Q)_{0} W\left(\zeta_{1}, \zeta_{2}\right) \exp \left(ß \lambda S\left(\zeta_{1}, \zeta_{2}\right)\right)$.

Here the phase function $S$ and the positive definite localization function $W$ are functions of the streamline invariants $\zeta_{1}$ and $\zeta_{2}$. The constant $\lambda$ is a large parameter. The effective wave number is

$\boldsymbol{k}=\lambda \nabla S$.
On a particular streamline with $\zeta_{1}=\zeta_{10}$ and $\zeta_{2}=\zeta_{20}$ say, this is a general linear combination of vectors in the $\nabla \zeta_{1}$ and $\nabla \zeta_{2}$ directions. These are independent because $\zeta_{1}$ and $\zeta_{2}$ are. The parameter $\lambda$ can be used to make the length scale $1 /|\boldsymbol{k}| \propto 1 / \lambda$ arbitrarily small. The localization function $W$ is chosen to approach zero as one moves away from a particular streamline on a length scale that approaches zero as $\lambda \rightarrow \infty$ but more slowly than $1 /|\boldsymbol{k}|$ say as $\propto 1 / \lambda^{1 / 2}$.

The amplitude factor $(\Delta Q)_{0}$ is assumed to be a function of time and slowly varying in space. Because of the localization this is effectively along the streamline with $\zeta_{1}=\zeta_{10}$ and $\zeta_{2}=\zeta_{20}$.

Adopting the above form of perturbations, because of the rapid variation of the complex phase $\lambda S$, one can make the standard assumption of local analysis that only the variation of this needs to be considered when taking spatial derivatives. The only exception occurs when the variation is only along streamlines. This happens when the derivatives in an expression occur in the form $\boldsymbol{v} \cdot \nabla$ as in for example Eqs. (22)-(23). Then, because $\boldsymbol{v} \cdot \nabla S=0$, the contribution $\boldsymbol{v} \cdot \nabla\left(\Delta Q_{0}\right)$ must be retained.

Proceeding in this way one may obtain a set of governing equations for quantities on a streamline on which the perturbation is localized. Because the exponential and localization factors cancel out we drop the subscript zero from the perturbation amplitudes. Apart from such a justification as in standard local analysis one can also prove that the eigenvalues $\sigma$ one obtains belong to the spectrum of the global linear stability problem (see Appendix C; and also Papaloizou \& Pringle 1982; Terquem \& Papaloizou 1996, for a discussion of related problems).

\subsection{Reduction of the linearized equations}

Before embarking on a local analysis, we perform a prior reduction of the stability problem to a convenient form without approximation.

We note that

$\frac{D^{2} \boldsymbol{\xi}}{D t^{2}}+2 \mathbf{\Omega}_{\boldsymbol{P}} \times \frac{D \boldsymbol{\xi}}{D t}=\boldsymbol{a}$.

$a=\Delta \boldsymbol{F}-\mathbf{\Omega}_{P} \times\left(\mathbf{\Omega}_{P} \times \boldsymbol{\xi}\right)$.

Using Eqs. (4) and (19)-(21), after some algebra, we can express $\boldsymbol{a}$ in the form

$\boldsymbol{a}=-\frac{H}{\rho} \nabla\left(\frac{P^{\prime}}{H}\right)+\frac{\nabla P}{\rho} \xi \cdot\left(\frac{\nabla P}{\Gamma P}-\frac{\nabla \rho}{\rho}\right)$

$+\boldsymbol{\xi} \cdot \nabla\left(\boldsymbol{v} \cdot \nabla \boldsymbol{v}+2 \mathbf{\Omega}_{P} \times \boldsymbol{v}\right)$,

where $H=P^{1 / \Gamma}$.

Using Eqs. (19)-(21) we may also write

$P^{\prime}=-\frac{\Gamma P}{H} \nabla(\cdot \xi H)$.

In what follows we find it convenient to decompose $\boldsymbol{\xi} H$ into solenoidal and irrotational parts such that

$\boldsymbol{\xi} H=\frac{\nabla \times \mathbf{A}}{\lambda}+\frac{\nabla \phi}{\lambda^{3}}$ 
The powers of the large parameter $\lambda$ are introduced so as to anticipate the order of terms of different types. Thus it will turn out that $\boldsymbol{\xi} H$ is mainly solenoidal with an irrotational correction smaller in magnitude by a factor of order $\lambda^{-2}$. From Eq. (31) it then follows that

$P^{\prime}=-\frac{\Gamma P \nabla^{2} \phi}{H \lambda^{3}}$

and

$$
\begin{aligned}
\boldsymbol{a}= & \frac{H}{\rho} \nabla\left(\frac{\Gamma P \nabla^{2} \phi}{H^{2} \lambda^{3}}\right)+\frac{\nabla P}{\rho} \boldsymbol{\xi} \cdot\left(\frac{\nabla P}{\Gamma P}-\frac{\nabla \rho}{\rho}\right) \\
& +\boldsymbol{\xi} \cdot \nabla\left(\boldsymbol{v} \cdot \nabla \boldsymbol{v}+2 \mathbf{\Omega}_{\boldsymbol{P}} \times \boldsymbol{v}\right) .
\end{aligned}
$$

\subsubsection{Effect of the equation of state}

We comment here that the above analysis assumed an adiabatic condition for which the stability problem has a self-adjoint character (e.g. Lynden-Bell \& Ostriker 1967). However, because of the local nature of the formalism considered here we may also consider other equations of state for which the formal self-adjoint character does not hold in the fully global problem while it is recovered in the local problem (see Sect. 7).

In particular, with a later application to disk configurations in mind we consider equations of state of the form $P=\rho c^{2}(\boldsymbol{r})$. Here $c$ is the sound speed which is specified to be an arbitrary function of $\boldsymbol{r}$. The equation of state also holds for perturbations so that $P^{\prime}=\rho^{\prime} c^{2}$.

Then in place of Eq. (31) we have

$P^{\prime}=-c^{2} \nabla \cdot(\rho \xi)$.

This is of the same form if we identify $H=\rho$, and $\Gamma P=H c^{2}$. With this identification we now get instead of Eq. (30) that

$\boldsymbol{a}=-\frac{H c^{2}}{\rho} \nabla\left(\frac{P^{\prime}}{H c^{2}}\right)+\boldsymbol{\xi} \cdot \nabla\left(\boldsymbol{v} \cdot \nabla \boldsymbol{v}+2 \mathbf{\Omega}_{P} \times \boldsymbol{v}\right)$.

This differs by the positioning of $c^{2}$ and the the absence of the second entropy gradient term. However, the positioning of $c^{2}$ is immaterial for the local analysis presented (it may be taken through the differentiation) and thus the formalism is the same as in the adiabatic equation of state case when the unperturbed entropy is constant or equivalently $\nabla P /(\Gamma P)=\nabla \rho / \rho$.

\subsection{Solution procedure in the local limit}

Although we introduced the small parameter $\lambda^{-1}$, above we have not yet used this smallness to simplify the stability problem. We here reduce the Eq. (28) together with Eq. (34) to leading order in $\lambda^{-1}$, which is here equivalent to taking the limit $\lambda^{-1} \rightarrow 0$.

We begin by noting that to leading order in $\lambda^{-1}, \xi H$ is solenoidal $(\nabla \cdot(\xi H)=0)$ as follows from considering the right hand side of Eq. (32).

To specify a form for $\boldsymbol{\xi} H$ that is solenoidal to leading order in $\lambda^{-1}$, we note that to this order only variations of $S$ need to be considered (see Sect. 4.2) so that this is equivalent to requiring that $\boldsymbol{k} \cdot \boldsymbol{\xi}=0$.
In this case $\xi$ may be written as a linear combination of two linearly independent unit vectors $\boldsymbol{b}_{1}$ and $\boldsymbol{b}_{2}$ that are perpendicular to $\boldsymbol{k} \propto \nabla S$.

To specify two such unit vectors we first use the fact that $\boldsymbol{v} \cdot \nabla S=0$ and adopt

$\boldsymbol{b}_{1}=\frac{\boldsymbol{v}}{|\boldsymbol{v}|}$.

We can then adopt

$\boldsymbol{b}_{2}=\frac{\boldsymbol{v} \times \nabla S}{|\boldsymbol{v} \times \nabla S|}$

which gives a unit vector orthogonal to both $\boldsymbol{v}$ and $\boldsymbol{k}$.

In adopting the above basis we make the assumption that there are no stagnation points in the flow at which $|\boldsymbol{v}|=0$.

In this case may then adopt the decomposition

$\boldsymbol{\xi}=\alpha \boldsymbol{b}_{1}+\beta \boldsymbol{b}_{2}$

where $\alpha$ and $\beta$ are of the general form given by Eq. (26).

The solution strategy is then to substitute the form of the displacement (39) into Eq. (28) together with Eq. (34), to take the scalar product of the result with respect to $\boldsymbol{b}_{1}$ and $\boldsymbol{b}_{2}$ respectively. This results in two equations to determine $\alpha$ and $\beta$ on the streamline on which the perturbation is localized.

Noting that as the dependence on $\zeta_{1}$ and $\zeta_{2}$ can be eventually factored out and dropped and only convective derivatives are involved, in a Lagrangian description we can ultimately regard $\alpha$ and $\beta$ as functions only of time on a fixed streamline. Accordingly, these form a pair of coupled ordinary differential equations.

We begin by applying this procedure ro the left hand side of Eq. (28) which yields after straightforward algebra:

$\ddot{\alpha}+2 C_{0} \dot{\beta}+C_{11} \alpha+C_{12} \beta=\boldsymbol{a} \cdot \boldsymbol{b}_{1}$

and

$\ddot{\beta}-2 C_{0} \dot{\alpha}+C_{21} \alpha+C_{22} \beta=\boldsymbol{a} \cdot \boldsymbol{b}_{2}$.

Here in order to condense the notation, we set for the first and second order convective derivatives operating on any quantity $Q$

$\frac{D Q}{D t} \equiv \dot{Q}$

and

$\frac{D^{2} Q}{D t^{2}} \equiv \ddot{Q}$

It is particularly useful to recall that for time independent quantities such as the unit vectors introduced above

$\frac{D Q}{D t}=\boldsymbol{v} \cdot \nabla Q$.

We further have

$C_{0}=\dot{\boldsymbol{b}}_{2} \cdot \boldsymbol{b}_{1}+\left(\boldsymbol{\Omega}_{\boldsymbol{P}} \times \boldsymbol{b}_{2}\right) \cdot \boldsymbol{b}_{1}=-\dot{\boldsymbol{b}}_{1} \cdot \boldsymbol{b}_{2}-\left(\boldsymbol{\Omega}_{\boldsymbol{P}} \times \boldsymbol{b}_{1}\right) \cdot \boldsymbol{b}_{2}$,

and for $i=1,2$ and $j=1,2$

$C_{i j}=\ddot{\boldsymbol{b}}_{j} \cdot \boldsymbol{b}_{i}+\left(2 \boldsymbol{\Omega}_{\boldsymbol{P}} \times \dot{\boldsymbol{b}}_{j}\right) \cdot \boldsymbol{b}_{i}$. 
Using Eq. (34) we can find $\boldsymbol{a} \cdot \boldsymbol{b}_{1}$, and $\boldsymbol{a} \cdot \boldsymbol{b}_{2}$ which occur on the right hand sides of Eqs. (40) and (40) respectively.

We begin by noting that in the limit $\lambda^{-1} \rightarrow 0, \nabla$ reduces to multiplication by $i \lambda \nabla S$. Thus in this limit

$$
\begin{aligned}
\boldsymbol{a}= & -i\left(\frac{\Gamma P|\nabla S|^{2} \nabla S}{\rho H}\right) \phi+\frac{\nabla P}{\rho} \boldsymbol{\xi} \cdot\left(\frac{\nabla P}{\Gamma P}-\frac{\nabla \rho}{\rho}\right) \\
& +\boldsymbol{\xi} \cdot \nabla\left(\boldsymbol{v} \cdot \nabla \boldsymbol{v}+2 \mathbf{\Omega}_{\boldsymbol{P}} \times \boldsymbol{v}\right),
\end{aligned}
$$

with the form of $\boldsymbol{\xi}$ being given by Eq. (39).

Using the above we find

$$
\boldsymbol{a} \cdot \boldsymbol{b}_{1}=D_{11} \alpha+D_{12} \beta
$$

and

$\boldsymbol{a} \cdot \boldsymbol{b}_{2}=D_{21} \alpha+D_{22} \beta$,

where for $i=1,2$ and $i=1,2$

$$
\begin{aligned}
D_{i j}= & \boldsymbol{b}_{i} \cdot\left(\boldsymbol{b}_{j} \cdot \nabla\left(\boldsymbol{v} \cdot \nabla \boldsymbol{v}+2 \boldsymbol{\Omega}_{\boldsymbol{P}} \times \boldsymbol{v}\right)\right) \\
& +\boldsymbol{b}_{i} \cdot \frac{\nabla P}{\rho} \boldsymbol{b}_{j} \cdot\left(\frac{\nabla P}{\Gamma P}-\frac{\nabla \rho}{\rho}\right) .
\end{aligned}
$$

Thus the governing equations for $\alpha$ and $\beta$ may be cast in the final form

$\ddot{\alpha}+2 C_{0} \dot{\beta}=E_{11} \alpha+E_{12} \beta$

and

$\ddot{\beta}-2 C_{0} \dot{\alpha}=E_{21} \alpha+E_{22} \beta$,

where $E_{i, j}=D_{i, j}-C_{i, j}$.

To complete the solution the scalar $\phi$ may be determined by taking the scalar product of Eq. (28) with $\boldsymbol{k}$. However, this is not needed for our purposes so we do not consider it further here.

We comment that the following identities, valid for any unit vectors, are useful for evaluating the coefficients in the governing equations for $\alpha$ and $\beta$ that involve second time derivatives:

$\ddot{\boldsymbol{b}}_{i} \cdot \boldsymbol{b}_{i}=-\left|\dot{\boldsymbol{b}}_{i}\right|^{2}$

and

$\ddot{\boldsymbol{b}}_{i} \cdot \boldsymbol{b}_{j}=-\dot{\boldsymbol{b}}_{i} \cdot \dot{\boldsymbol{b}}_{j}+\frac{D\left(\dot{\boldsymbol{b}}_{i} \cdot \boldsymbol{b}_{j}\right)}{D t}$.

The governing Eqs. (51) and (52) consist of a pair of second order differential equations for the amplitudes $(\alpha, \beta)$ as a function of time. As the above is a Lagrangian description the coefficients appearing in these equations generically are periodic functions of time. This is because these coefficients describe fluid effects as seen by a fluid element as it circulates periodically on an unperturbed streamline. Only when these conditions are invariant such as on circular streamlines in a system with cylindrical symmetry are the coefficients constant. In the generic case the periodicity of the coefficients leads to a system similar to the well known cases of Mathieu's or Hill's equation (e.g. Whittaker \& Watson 1996). Accordingly we expect to encounter parametric instability when a normal mode frequency is commensurable with the orbital frequency associated with circulating around a streamline. The lowest order relation is when the normal mode frequency is one half of the orbital frequency. This is easy to demonstrate when the system is almost cylindrically symmetric and the streamlines depart from circles by a small amount (see below and Appendix B). Furthermore commensurability conditions can always be satisfied when the underlying modes are gravity/inertial modes because, as is well known, their spectrum is dense (e.g. Papaloizou \& Pringle 1982; Lin et al. 1993). This density comes about because in the local limit the oscillation frequencies depend only on wavenumber ratios so that wavenumber components may be allowed to tend to infinity at fixed ratios while not affecting the oscillation frequency. One can use that property to relate the spectrum of the local problem to that of the global problem (see Appendix C). We illustrate the above remarks by considering a system with cylindrical symmetry and a system for which the streamlines are Keplerian ellipses of arbitrary eccentricity. Of course in the latter case when the eccentricity is small the streamlines deviate slightly from circles.

\section{The case of circular streamlines in the steady state}

In this case, $\boldsymbol{\Omega}_{\boldsymbol{P}}=0$, and the invariants on a streamline are taken to be $\zeta_{1}=r$, and $\zeta_{2}=z$. Because the orbits are circles we have $r=a$, where $a$ is the semi-major axis.

For the phase function we adopt $S=K_{r} r+K_{z} z$ with $K_{r}$ and $K_{z}$ being constants.

This gives the wavenumber $\boldsymbol{k}=\lambda \nabla S=\left(\lambda K_{r}, 0, \lambda K_{z}\right)$.

For this steady state model $v=\left(0, v_{\varphi}(r), 0\right)$, Eq. (37) gives $\boldsymbol{b}_{1}=\hat{\boldsymbol{\varphi}}$ being the unit vector in the azimuthal direction. Then from Eq. (38) we obtain $\boldsymbol{b}_{2}=\left(K_{z} \hat{\boldsymbol{r}}-K_{r} \hat{\boldsymbol{k}}\right) / K$, where $\hat{\boldsymbol{r}}$ and $\hat{\boldsymbol{k}}$ are unit vectors in the radial and vertical directions respectively and $K=\sqrt{K_{r}^{2}+K_{z}^{2}}$.

Having obtained these unit vectors the forms of Eqs. (51) and (52) can be found by direct calculation.

Using $D \hat{\boldsymbol{r}} / D t=\boldsymbol{v} \cdot \nabla \hat{\boldsymbol{r}}=v_{\varphi} \hat{\boldsymbol{\varphi}} / r$, and $D \hat{\boldsymbol{\varphi}} / D t=\boldsymbol{v} \cdot \nabla \hat{\boldsymbol{\varphi}}=$ $-v_{\varphi} \hat{\boldsymbol{r}} / r$, we find setting $\Omega=v_{\varphi} / r$ which is constant on streamlines, that

$\frac{D \boldsymbol{b}_{1}}{D t}=-\Omega \hat{\boldsymbol{r}}$

and

$\frac{D \boldsymbol{b}_{2}}{D t}=\frac{K_{z} \Omega \hat{\varphi}}{K}$

From these it is easy to obtain $C_{0}=\boldsymbol{b}_{1} \cdot \dot{\boldsymbol{b}}_{2}=K_{z} \Omega / K, \ddot{\boldsymbol{b}}_{1} \cdot \boldsymbol{b}_{2}=$ $\ddot{\boldsymbol{b}}_{2} \cdot \boldsymbol{b}_{1}=0, \ddot{\boldsymbol{b}}_{1} \cdot \boldsymbol{b}_{1}=-\Omega^{2}$, and $\ddot{\boldsymbol{b}}_{2} \cdot \boldsymbol{b}_{2}=-K_{z}^{2} \Omega^{2} / K^{2}$.

Further we find that for $\xi$ given by Eq. (39) that

$\boldsymbol{b}_{1} \cdot(\boldsymbol{\xi} \cdot \nabla(\boldsymbol{v} \cdot \nabla \boldsymbol{v}))=-\Omega^{2} \alpha$,

and

$\boldsymbol{b}_{2} \cdot(\boldsymbol{\xi} \cdot \nabla(\boldsymbol{v} \cdot \nabla \boldsymbol{v}))=-\frac{\Omega^{2} \beta K_{z}^{2}}{K^{2}}-\frac{2 \Omega r \Omega^{\prime} \beta K_{z}^{2}}{K^{2}}$. 
In addition for $i=1,2, j=1,2$,

$$
\begin{aligned}
\boldsymbol{b}_{i} \cdot \frac{\nabla P}{\rho} \boldsymbol{b}_{j} \cdot & \left(\frac{\nabla P}{\Gamma P}-\frac{\nabla \rho}{\rho}\right)= \\
& \frac{\hat{\boldsymbol{\varphi}} \cdot(\boldsymbol{k} \times \nabla P)}{|\boldsymbol{k}| \rho} \frac{\hat{\boldsymbol{\varphi}} \cdot\left(\boldsymbol{k} \times\left(\frac{\nabla P}{\Gamma P}-\frac{\nabla \rho}{\rho}\right)\right)}{|\boldsymbol{k}|} \delta_{i 2} \delta_{j 2},
\end{aligned}
$$

with $\delta_{i j}$ denoting the Kronnecker delta.

Using Eqs. (57)-(59) and above, Eqs. (51) and (52) are reduced to the simple forms

$\ddot{\alpha}+\frac{2 K_{z} \Omega}{K} \dot{\beta}=0$

$\ddot{\beta}-\frac{2 K_{z} \Omega}{K} \dot{\alpha}=-\left(\frac{2 \Omega r \Omega^{\prime} K_{z}^{2}}{K^{2}}+v^{2}\right) \beta$,

where

$v^{2} K^{2}=-\hat{\boldsymbol{\varphi}} \cdot\left(\boldsymbol{k} \times\left(\frac{\nabla P}{\rho}\right)\right) \hat{\boldsymbol{\varphi}} \cdot\left(\boldsymbol{k} \times\left(\frac{\nabla P}{\Gamma P}-\frac{\nabla \rho}{\rho}\right)\right)$.

In this case because there is no variation of state variables around streamlines the governing equations have constant coefficients and we may look for normal mode solutions for which $\alpha$ and $\beta$ are $\alpha \exp (i \sigma t)$ where $\sigma$ is the normal mode frequency.

Setting $\alpha=\alpha_{0} \exp (i \sigma t), \beta=\beta_{0} \exp (i \sigma t)$ Eqs. (60)-(62) give a non trivial solution such that

$\alpha=\frac{2 i K_{z} \Omega}{K \sigma} \beta$

and

$\sigma^{2}=\frac{2 \Omega\left(r \Omega^{\prime}+2 \Omega\right) K_{z}^{2}}{K^{2}}+v^{2}$.

This gives the well known local dispersion for inertial/gravity modes in a differentially rotating system (see e.g. Tassoul 1978, and references therein for a discussion). There are two contributions. The first is from the rotation and depends on the gradient of angular momentum which must be positive for stability (Rayleigh's criterion) and the second is from the entropy gradient and leads to gravity waves when stable $\left(v^{2}>0\right)$ or convection when convectively unstable (Schwarzchild's criterion). We shall always assume that the system is stable to convection and satisfies Rayleigh's criterion. Accordingly we consider systems that are dynamically stable in this sense.

However, as we shall see below parametric instability can occur when state variables vary around streamlines making a perturbed fluid element subject to periodic restoring forces even though the system is stable according to the Rayleigh and Schwarzchild criteria.

An important feature in this context is that the oscillation spectrum is dense. This is because the local dispersion relation depends only on the wavenumber ratio $K_{r} / K_{z}$. As in a local analysis both $\lambda K_{r}$ and $\lambda K_{z} \rightarrow \infty$ these may be adjusted to make the ratio take on any finite value and thus produce a continuous or everywhere dense spectrum (see e.g. Papaloizou \& Pringle 1982; Lin et al. 1993, for a discussion of this point in a global context).
This property of the spectrum means that commensurability conditions such as $\sigma=\Omega / 2$, with $\Omega$ being the angular frequency associated with one orbit around a streamline, required for parametric instability can always be adequately satisfied.

For example Eq. (64) gives for inertial modes $\left(v^{2}=0\right)$ and Keplerian rotation, $\sigma^{2}=\Omega^{2} K_{z}^{2} / K^{2}$. Thus when $K_{z} / K_{r}=1 / \sqrt{3}$, $\sigma= \pm \Omega / 2$.

Suppose, the radial and vertical wavenumber components have to be integral multiples of some smallest values $\left(K_{r 0}, K_{z 0}\right)$ respectively. Then because we can let both wavenumber components become arbitrarily large, and because of the ability to approximate irrationals arbitrarily closely with rationals, we can make $\left|K_{z} / K_{r}-\sqrt{3}\right|$ become as small as we like.

\section{A steady state model with streamlines being Keplerian ellipses}

We now consider a more interesting non axisymmetric model for which the streamlines correspond to Keplerian ellipses with the same eccentricity circulating about a central point mass. This is described in Sect. 3. In this particular model the streamlines form a pattern that is stationary in the inertial frame. However, in more general cases the pattern may precess slowly and so appear stationary only in a slowly rotating frame. But this effect should make little difference. We comment also that because we are considering local stability, where perturbations are localized along streamlines, these streamlines are only required to fill a small local volume of the fluid independently of exterior conditions.

In this model fluid state variables vary as a fluid element circulates around a streamline. Thus periodic forcing of a perturbed fluid element occurs and parametric instability becomes possible.

The velocity field for the streamlines was specified through Eqs. (7) and (8) in Sect. 3. To proceed we need to specify two invariants for the streamlines. Because for the cylindrical disk model adopted, the motion is in horizontal planes so one of these may be taken to be $z$. The other may be taken to be $\left.a\left(1-e^{2}\right)=r(1+\cos \varphi)\right)$ which is proportional to the square of the angular momentum.

Using these we adopt for the phase function

$S=K_{r} r(1+e \cos \varphi)+K_{z} z$ with $K_{r}$ and $K_{z}$ again being constants.

The wavenumber is then given by

$\boldsymbol{k}=\lambda \nabla S=\left(\lambda K_{r}(1+e \cos \varphi),-\lambda K_{r} e \sin \varphi, \lambda K_{z}\right)$.

In this case

$v=\left(\sqrt{\frac{G M_{*}}{r(1+e \cos \varphi)}} e \sin \varphi, \sqrt{\frac{G M_{*}(1+e \cos \varphi)}{r}}, 0\right)$,

so that Eq. (37) gives

$\boldsymbol{b}_{1}=\frac{\boldsymbol{v}}{|\boldsymbol{v}|}=\frac{e \sin \varphi \hat{\boldsymbol{r}}+(1+e \cos \varphi) \hat{\varphi}}{\sqrt{\left(1+e^{2}+2 e \cos \varphi\right)}}$ 
From Eq. (38) we obtain

$$
\begin{aligned}
\boldsymbol{b}_{2}= & \frac{K_{z}(1+e \cos \varphi) \hat{\boldsymbol{r}}-K_{z} e \sin \varphi \hat{\boldsymbol{\varphi}}}{N \sqrt{\left(1+e^{2}+2 e \cos \varphi\right)}} \\
& -\frac{K_{r} \sqrt{\left(1+e^{2}+2 e \cos \varphi\right)}}{N},
\end{aligned}
$$

where $\hat{\boldsymbol{k}}$ is the unit vector in the vertical direction and $N=$ $\sqrt{K_{r}^{2}\left(1+e^{2}+2 e \cos \varphi\right)+K_{z}^{2}}$ is the normalizing factor required to ensure that $\boldsymbol{b}_{2}$ is a unit vector.

Given these unit vectors the forms of Eqs. (51) and (52) can again be found by direct calculation. As the details are somewhat tedious they are relegated to Appendix A.

The coefficients $\left(E_{i, j}, i=1,2, j=1,2, C_{0}\right)$ contained in the linear governing Eqs. (51) and (52) for $\alpha$ and $\beta$ are given by

$$
\begin{aligned}
E_{11}= & -\frac{G M_{*}}{r^{3}} \frac{\left(1+2 e \cos \varphi+e^{2}\left(1-3 \sin ^{2} \varphi\right)\right)}{1+e^{2}+2 e \cos \varphi} \\
& +\frac{G M_{*}}{r^{3}} \frac{(1+e \cos \varphi)^{3}}{\left(1+e^{2}+2 e \cos \varphi\right)^{2}}, \\
E_{12}= & \frac{G M_{*}}{r^{3}} \frac{3 K_{z} e \sin \varphi(1+e \cos \varphi)}{N\left(1+e^{2}+2 e \cos \varphi\right)}+\dot{\boldsymbol{b}}_{1} \cdot \dot{\boldsymbol{b}}_{2}-\frac{D C_{0}}{D t}, \\
E_{21}= & \frac{G M_{*}}{r^{3}} \frac{3 K_{z} e \sin \varphi(1+e \cos \varphi)}{N\left(1+e^{2}+2 e \cos \varphi\right)}+\dot{\boldsymbol{b}}_{1} \cdot \dot{\boldsymbol{b}}_{2}+\frac{D C_{0}}{D t}
\end{aligned}
$$

and

$E_{22}=\frac{G M_{*}}{r^{3}} \frac{K_{z}^{2}\left(2+4 e \cos \varphi+e^{2}\left(2-3 \sin ^{2} \varphi\right)\right)}{N^{2}\left(1+e^{2}+2 e \cos \varphi\right)}-\boldsymbol{b}_{2} \ddot{\boldsymbol{b}}_{2}$.

In addition

$C_{0}=\boldsymbol{b}_{1} \cdot \dot{\boldsymbol{b}}_{2}=\sqrt{\frac{G M_{*}}{r^{3}}} \frac{K_{z}(1+e \cos \varphi)^{3 / 2}}{N\left(1+e^{2}+2 e \cos \varphi\right)}$

and

$$
\begin{aligned}
\frac{D C_{0}}{D t}= & -\frac{G M_{*}}{r^{3}} \frac{K_{z}(1+e \cos \varphi) e \sin \varphi}{N\left(1+e^{2}+2 e \cos \varphi\right)} \\
& \times\left(\frac{\left(1+3 e^{2}+4 e \cos \varphi\right)}{\left(1+e^{2}+2 e \cos \varphi\right)}-\frac{K_{r}^{2}(1+e \cos \varphi)}{N^{2}}\right)
\end{aligned}
$$

with

$$
\frac{D N}{D t}=-\sqrt{\frac{G M_{*}}{r^{3}}} \frac{K_{r}^{2} e \sin \varphi \sqrt{1+e \cos \varphi}}{\sqrt{K_{z}^{2}+K_{r}^{2}\left(1+e^{2}+2 e \cos \varphi\right)}} .
$$

We also have

$$
\begin{aligned}
\ddot{\boldsymbol{b}}_{2} \cdot \boldsymbol{b}_{2}= & -\frac{G M_{*}(1+e \cos \varphi)}{r^{3}} \\
& \times\left(\frac{(1+e \cos \varphi)^{2} K_{z}^{2}+K_{r}^{2} e^{2} \sin ^{2} \varphi\left(1+e^{2}+2 e \cos \varphi\right)}{N^{2}\left(1+e^{2}+2 e \cos \varphi\right)^{2}}\right. \\
& \left.-\frac{K_{r}^{4} e^{2} \sin ^{2} \varphi}{N^{2}\left(K_{z}^{2}+K_{r}^{2}\left(1+e^{2}+2 e \cos \varphi\right)\right)}\right) .
\end{aligned}
$$

together with

$\dot{\boldsymbol{b}}_{2} \cdot \dot{\boldsymbol{b}}_{1}=\sqrt{\frac{G M_{*}}{r^{3}}} \frac{D N}{D t} \frac{K_{z}(1+e \cos \varphi)^{3 / 2}}{N^{2}\left(1+e^{2}+2 e \cos \varphi\right)}$,

which enables all the coefficients to be calculated.

\section{Solution of the governing equations}

We first note that the governing Eqs. (51) and (52) can be written in a compact vector form with similar structure to the Lynden-Bell \& Ostriker (1967) formulation of the general stability problem (see Eqs. (18) and (24)):

$\ddot{\boldsymbol{y}}-2 C_{0} \boldsymbol{e}_{3} \times \dot{\boldsymbol{y}}=\boldsymbol{E} \cdot \boldsymbol{y}$.

Here the two dimensional vector $\boldsymbol{y}^{T}=(\alpha, \beta)$ and the vector $\boldsymbol{e}_{3} \times \dot{\boldsymbol{y}}=(-\dot{\beta}, \dot{\alpha})^{T}$. The two dimensional matrix $\boldsymbol{E}$ has elements $E_{11}, E_{12}, E_{21}, E_{22}$ as defined in Sect. 4.4 above.

When a system has stream lines perturbed slightly from circles and the unperturbed circular streamline case has an underlying spectrum of oscillation modes with a dense spectrum, it is possible to show analytically that the system has a generic parametric instability. This is done in Appendix B.

However, we have found that the most direct method of assessing the stability of a general system, with streamlines differing significantly from circles, governed by Eq. (77) is to integrate the system as an initial value problem starting from general initial conditions and to examine whether the solutions manifest exponential growth (see below). In this way, which is effectively exploiting the Lagrangian nature of the system and solving for conditions in the neighbourhood of a fluid element as it circulates around streamline, problems with boundary conditions are avoided.

However, before presenting an example we briefly comment that the system (77) can be dealt with in an equivalent Eulerian formulation in which an eigenvalue problem of the same type as that in the Lynden-Bell \& Ostriker formalism is recovered. To obtain the Eulerian formulation the convective derivatives in Eq. (77) are replaced as in (15). Thus

$\frac{D \boldsymbol{y}}{D t}=\frac{\partial \boldsymbol{y}}{\partial t}+\boldsymbol{v} \cdot \nabla \boldsymbol{y}$.

As our system has spatial variation only around streamlines, we can write $\boldsymbol{v} \cdot \nabla \boldsymbol{y}=|\boldsymbol{v}| \mathrm{d} \boldsymbol{y} / \mathrm{d} s$, with $s$ measuring the arc length along a streamline. The problem is thus a one dimensional one with periodic boundary conditions in $s$. One now adopts a time dependence through a factor $\exp (\beta \sigma t)$ and obtains an eigenvalue problem for $\sigma$ of the form (24) namely

$-\sigma^{2} \boldsymbol{y}+\sigma \mathcal{B}(\boldsymbol{y})+C(\boldsymbol{y})=0$,

where each of the operators $\mathcal{B}, C$ is self adjoint with the inner product for general vectors $\boldsymbol{y}_{1}, \boldsymbol{y}_{2}$ defined as

$\left(\boldsymbol{y}_{2}, \boldsymbol{y}_{1}\right)=\int \rho \boldsymbol{y}_{2}^{*} \cdot \boldsymbol{y}_{1} \mathcal{A d} s$,

where $\mathcal{A}$ is the stream tube area function (see Sect. 3.2) and the integral is taken around the streamline of interest.

This formulation allows us to note some of the results of Lynden-Bell \& Ostriker (1967) such as that eigenvalues occur in quartets with complex conjugates and with opposite signs.

\subsection{Numerical solution for Kepler ellipses}

We have solved the governing equations (see Eq. (77) in Sect. 7) numerically as an initial value problem. To do this 


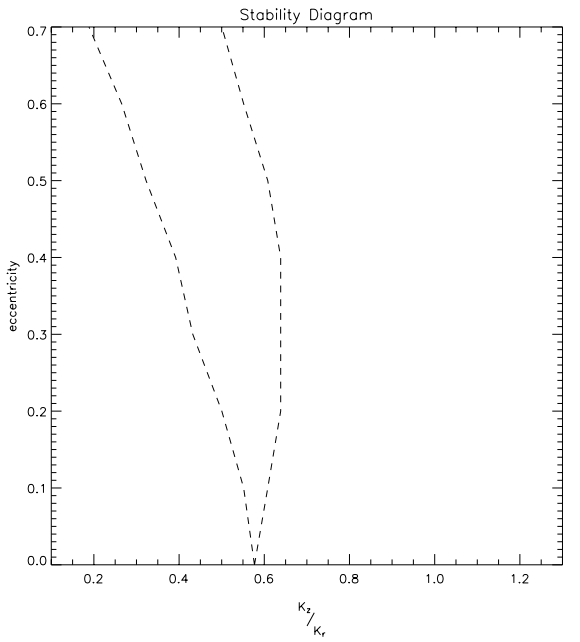

Fig. 1. The stability diagram for Eqs. (51) and (52) applied to the model with streamlines which are Keplerian ellipses in the $\left(\frac{K_{z}}{K_{r}}, e\right)$ plane. Instability occurs in the region of the plane between the two curves plotted.

we specify the ratio $K_{z} / K_{r}$ and integrate the system forward in time from arbitrary initial conditions. Instability then shows up as exponential growth, the rate may be easily estimated in practice. This procedure is very much simpler than attempting to solve a matrix eigenvalue problem.

In the limit of small eccentricity the problem can be solved analytically using a standard approach to parametric instability problems. This is done in Appendix B. In this case the maximum instability occurs when $K_{z} / K_{r}=1 / \sqrt{3}$. At this wavenumber ratio, the maximum growth rate is

$\lambda=3 e \Omega / 16$.

The calculation is a Lagrangian one so viewed from the point of view of a fluid element circulating around a streamline, the instability occurs through the interaction of two inertial modes with frequencies $\pm \Omega / 2$. In an Eulerian sense, if the pattern is stationary in the inertial frame, the frequencies as viewed there are $-m_{1} \Omega+\Omega / 2$ and $-m_{2} \Omega-\Omega / 2$, where $\left(m_{1}, m_{2}\right)$ are the associated azimuthal mode numbers. These frequencies must be equal in the stationary frame because there is only one eigenfrequency $\sigma$ as viewed from there as considered above. This means that $m_{1}-m_{2}=1$. Otherwise the $m_{i}$ are not constrained.

Numerical solution of the governing equations gives the stability diagram plotted in Fig. 1. This shows the region of the $\left(K_{z} / K_{r}, e\right)$ plane with instability for $0<e<0.7$. When $e \rightarrow 0$, the numerical and analytic results agree. The region of instability collapses onto $K_{z} / K_{r}=1 / \sqrt{3}$ and the maximum growth rate agrees with Eq. (81). The band of instability increases with $e$.

The Maximum growth rate in units of $\sqrt{G M_{*} / a^{3}}$ is plotted as a function of eccentricity in Fig. 2. This indicates that the maximum growth rate for a given $e$, is such that, to a good approximation, as in the small $e$ limit, $\lambda \propto e$ for $e<0.7$.

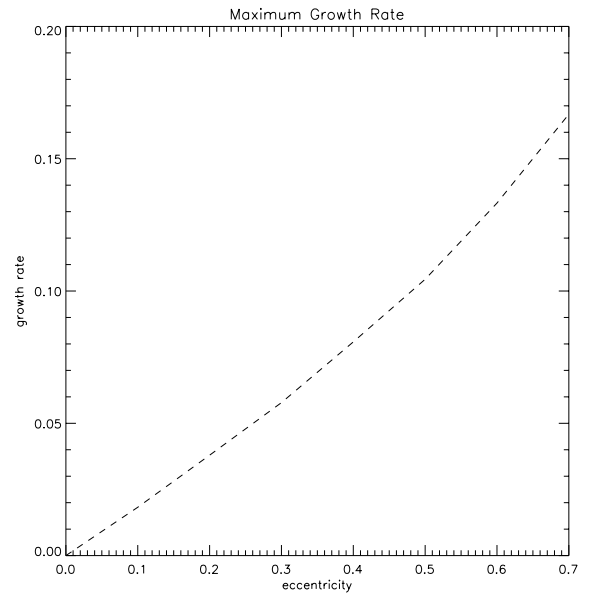

Fig. 2. Maximum growth rate in units of $\sqrt{G M_{*} / a^{3}}$ plotted as a function of eccentricity for solutions of Eqs. (51) and (52) applied to the model with streamlines which are Keplerian ellipses.

\section{Discussion}

In this paper we have formulated a general local stability analysis applicable to astrophysical flows that appear steady in an appropriate uniformly rotating coordinate system. Such a flow might correspond to a stellar mode of oscillation that is a travelling wave in the azimuthal direction or an accretion disk with a free eccentricity. In the simple example we considered the disk streamlines were Keplerian ellipses.

The general stability analysis considered perturbations localized in the neighbourhood of unperturbed fluid streamlines. The spatial localization was assumed to tend to zero as the wavenumber tended to infinity but more slowly than the inverse magnitude of the wavenumber itself allowing the adoption of wave packets. It is possible to consider the limit of infinite wave number taken at finite oscillation eigenfrequency and obtain spectral properties from the local theory that apply to the global problem. The physical reason for this is that the degrees of freedom under consideration correspond to inertial or gravity modes whose frequency depends only on the ratio of wavenumber components. The group velocity thus approaches zero with localization in a way that ensures that localization may be maintained for arbitrarily long times. This justifies the local procedure.

We found that the local stability of fluid on a particular streamline was governed by a pair of second order differential equations, which in a Lagrangian description, evolved the perturbation associated with a particular fluid element as a function of time. This reduces the stability problem to an initial value problem rather than an equivalent eigenvalue problem obtained in an Eulerian representation.

When conditions do not vary around streamlines one obtains standard oscillation modes. However, in the more general case when conditions vary around streamlines the ordinary differential equations are linear with periodic coefficients and the system was shown to become generically parametrically unstable. The dense spectra of inertial or gravity modes ensures that resonance conditions may always be satisfied. 
We applied our considerations to study the stability of fluid for which the streamlines were in Keplerian ellipses with eccentricity up to 0.7 . We showed that for small $e$, the maximum instability occurs when $K_{z} / K_{r}=1 / \sqrt{3}$ and involves, in the Eulerian description, two modes with azimuthal mode number differing by unity in magnitude. Then the growth rate is

$$
\lambda=3 e \Omega / 16
$$

For large eccentricities, to a good approximation, this gives the maximum growth rate as a function of eccentricity. However, the band of unstable wavenumber ratios shifts (see Fig. 1).

Finally the considerations in this paper are based on linear theory and so they cannot determine the outcome of the instability. Results based on three dimensional numerical simulations for accretion disks with elliptical streamlines will be presented in a following publication.

\section{Appendix A: Keplerian streamline model}

We here consider the derivation of the coefficients in the linear governing equations for $\alpha$ and $\beta$ for the model with streamlines corresponding to Keplerian ellipses described in Sect. 6. The velocity field is given by Eqs. (7) and (8) and the two invariants on streamlines are taken to be $z$ and $\left.a\left(1-e^{2}\right)=r(1+\cos \varphi)\right)$ being proportional to the square of the angular momentum.

We here note that the well known orbit equation given above may be used to eliminate $r$ in the expressions given below so that the coefficients only depend on $\varphi$ and quantities constant on a streamline. Given the dependence of $\varphi$ on time, they may also be expressed as functions of time and quantities constant on a streamline.

The phase function is $S=K_{r} r(1+e \cos \varphi)+K_{z} z$ with $K_{r}$ and $K_{z}$ being constants.

The wavenumber is given by $\boldsymbol{k}=\lambda \nabla S=\left(\lambda K_{r}(1+\right.$ $\left.e \cos \varphi),-\lambda K_{r} e \sin \varphi, \lambda K_{z}\right)$.

In this case

$\boldsymbol{v}=\left(\sqrt{\frac{G M_{*}}{r(1+e \cos \varphi)}} e \sin \varphi, \sqrt{\frac{G M_{*}(1+e \cos \varphi)}{r}}, 0\right)$

so that Eq. (37) gives

$\boldsymbol{b}_{1}=\frac{\boldsymbol{v}}{|\boldsymbol{v}|}=\frac{e \sin \varphi \hat{\boldsymbol{r}}+(1+e \cos \varphi) \hat{\boldsymbol{\varphi}}}{\sqrt{\left(1+e^{2}+2 e \cos \varphi\right)}}$

From Eq. (38) we obtain

$$
\begin{aligned}
\boldsymbol{b}_{2}= & \frac{K_{z}(1+e \cos \varphi) \hat{\boldsymbol{r}}-K_{z} e \sin \varphi \hat{\boldsymbol{\varphi}}}{N \sqrt{\left(1+e^{2}+2 e \cos \varphi\right)}} \\
& -\frac{K_{r} \sqrt{\left(1+e^{2}+2 e \cos \varphi\right)} \hat{\boldsymbol{k}}}{N},
\end{aligned}
$$

where $\hat{\boldsymbol{k}}$ is the unit vector in the vertical direction and $N=$ $\sqrt{K_{r}^{2}\left(1+e^{2}+2 e \cos \varphi\right)+K_{z}^{2}}$ is the normalizing factor required to ensure that $\boldsymbol{b}_{2}$ is a unit vector.

Given these unit vectors the forms of Eqs. (51) and (52) can again be found by direct calculation.
For this purpose it is useful to note for this model that the operator

$$
\begin{aligned}
\frac{D}{D t} & \equiv \boldsymbol{v} \cdot \nabla \\
& =\sqrt{\frac{G M_{*}}{r^{3}}}\left(\sqrt{(1+e \cos \varphi)} \frac{\partial}{\partial \varphi}+\frac{r e \sin \varphi}{\sqrt{(1+e \cos \varphi)}} \frac{\partial}{\partial r}\right)
\end{aligned}
$$

and to recall that $\partial \hat{\boldsymbol{r}} / \partial \varphi=\hat{\boldsymbol{\varphi}}$ and $\partial \hat{\hat{\varphi}} / \partial \varphi=-\hat{\boldsymbol{r}}$, while the unit vectors have zero derivative with respect to $r$.

Applying this operator to Eq. (37) we obtain

$$
\begin{aligned}
\frac{D \boldsymbol{b}_{1}}{D t}= & \sqrt{\frac{G M_{*}}{r^{3}}} \frac{(1+e \cos \varphi)^{3 / 2}}{\left(1+e^{2}+2 e \cos \varphi\right)^{3 / 2}} \\
& \times(-(1+e \cos \varphi) \hat{\boldsymbol{r}}+e \sin \varphi \hat{\varphi})
\end{aligned}
$$

and applying it to Eq. (38) we get

$$
\begin{aligned}
\frac{D\left(N \boldsymbol{b}_{2}\right)}{D t}= & \sqrt{\frac{G M_{*}}{r^{3}}}\left(\frac{K_{z}(1+e \cos \varphi)^{3 / 2} e \sin \varphi}{\left(1+e^{2}+2 e \cos \varphi\right)^{3 / 2}} \hat{\boldsymbol{r}}\right. \\
& +\frac{K_{z}(1+e \cos \varphi)^{5 / 2}}{\left(1+e^{2}+2 e \cos \varphi\right)^{3 / 2}} \hat{\boldsymbol{\varphi}} \\
& \left.+\frac{K_{r}(1+e \cos \varphi)^{1 / 2} e \sin \varphi}{\sqrt{\left(1+e^{2}+2 e \cos \varphi\right)}} \hat{\boldsymbol{k}}\right)
\end{aligned}
$$

with

$$
\frac{D N}{D t}=-\sqrt{\frac{G M_{*}}{r^{3}}} \frac{K_{r}^{2} e \sin \varphi \sqrt{1+e \cos \varphi}}{\sqrt{K_{z}^{2}+K_{r}^{2}\left(1+e^{2}+2 e \cos \varphi\right)}}
$$

From the above relations we obtain

$C_{0}=\boldsymbol{b}_{1} \cdot \dot{\boldsymbol{b}}_{2}=\sqrt{\frac{G M_{*}}{r^{3}}} \frac{K_{z}(1+e \cos \varphi)^{3 / 2}}{N\left(1+e^{2}+2 e \cos \varphi\right)}$

$\ddot{\boldsymbol{b}}_{1} \cdot \boldsymbol{b}_{1}=-\left|\dot{\boldsymbol{b}}_{1}\right|^{2}=-\frac{G M_{*}}{r^{3}} \frac{(1+e \cos \varphi)^{3}}{\left(1+e^{2}+2 e \cos \varphi\right)^{2}}$,

and

$$
\begin{aligned}
\ddot{\boldsymbol{b}}_{2} \cdot \boldsymbol{b}_{2}= & -\left|\dot{\boldsymbol{b}}_{2}\right|^{2}=-\frac{G M_{*}(1+e \cos \varphi)}{r^{3}} \\
& \times\left(\frac{(1+e \cos \varphi)^{2} K_{z}^{2}+K_{r}^{2} e^{2} \sin ^{2} \varphi\left(1+e^{2}+2 e \cos \varphi\right)}{N^{2}\left(1+e^{2}+2 e \cos \varphi\right)^{2}}\right. \\
& \left.-\frac{K_{r}^{4} e^{2} \sin ^{2} \varphi}{N^{2}\left(K_{z}^{2}+K_{r}^{2}\left(1+e^{2}+2 e \cos \varphi\right)\right)}\right) .
\end{aligned}
$$

We now use the general results for any pair of unit vectors obtained from Eqs. (53) and (54) that

$\ddot{\boldsymbol{b}}_{2} \cdot \boldsymbol{b}_{1}=-\dot{\boldsymbol{b}}_{1} \cdot \dot{\boldsymbol{b}}_{2}+\frac{D C_{0}}{D t}$

and

$\ddot{\boldsymbol{b}}_{1} \cdot \boldsymbol{b}_{2}=-\dot{\boldsymbol{b}}_{2} \cdot \dot{\boldsymbol{b}}_{1}-\frac{D C_{0}}{D t}$

to obtain $\ddot{\boldsymbol{b}}_{2} \cdot \boldsymbol{b}_{1}$ and $\ddot{\boldsymbol{b}}_{1} \cdot \boldsymbol{b}_{2}$ which occur in the equations governing $\alpha$ and $\beta$. 

obtain

We first apply the convective derivative operator (A.8) to

$$
\begin{aligned}
\frac{D C_{0}}{D t}= & -\frac{G M_{*}}{r^{3}} \frac{K_{z}(1+e \cos \varphi) e \sin \varphi}{N\left(1+e^{2}+2 e \cos \varphi\right)} \\
& \times\left(\frac{\left(1+3 e^{2}+4 e \cos \varphi\right)}{\left(1+e^{2}+2 e \cos \varphi\right)}-\frac{K_{r}^{2}(1+e \cos \varphi)}{N^{2}}\right)
\end{aligned}
$$

and use Eqs. (A.5)-(A.7) to obtain

$\dot{\boldsymbol{b}}_{2} \cdot \dot{\boldsymbol{b}}_{1}=\sqrt{\frac{G M_{*}}{r^{3}}} \frac{D N}{D t} \frac{K_{z}(1+e \cos \varphi)^{3 / 2}}{N^{2}\left(1+e^{2}+2 e \cos \varphi\right)}$

which enables these quantities to be found directly.

For this model, because the velocity field is Keplerian, we have the very useful result that

$\boldsymbol{v} \cdot \nabla \boldsymbol{v}=-\frac{G M_{*}}{r^{2}} \hat{\boldsymbol{r}}$.

Using this together with $\boldsymbol{\xi}$ given by Eq. (39) we obtain

$$
\begin{aligned}
\boldsymbol{b}_{1} \cdot(\boldsymbol{\xi} \cdot \nabla(\boldsymbol{v} \cdot \nabla \boldsymbol{v}))= & \beta \frac{G M_{*}}{r^{3}} \frac{3 K_{z} e \sin \varphi(1+e \cos \varphi)}{N\left(1+e^{2}+2 e \cos \varphi\right)} \\
& -\alpha \frac{G M_{*}}{r^{3}} \frac{1+2 e \cos \varphi+e^{2}\left(1-3 \sin ^{2} \varphi\right)}{1+e^{2}+2 e \cos \varphi},
\end{aligned}
$$

and

$$
\begin{array}{r}
\boldsymbol{b}_{2} \cdot(\boldsymbol{\xi} \cdot \nabla(\boldsymbol{v} \cdot \nabla \boldsymbol{v}))=\alpha \frac{G M_{*}}{r^{3}} \frac{3 K_{z} e \sin \varphi(1+e \cos \varphi)}{N\left(1+e^{2}+2 e \cos \varphi\right)} \\
+\beta \frac{G M_{*}}{r^{3}} \frac{K_{z}^{2}\left(2+4 e \cos \varphi+e^{2}\left(2-3 \sin ^{2} \varphi\right)\right)}{N^{2}\left(1+e^{2}+2 e \cos \varphi\right)} .
\end{array}
$$

In addition for this particular model with uniform pressure, we automatically have for $i=1,2, j=1,2$,

$\boldsymbol{b}_{i} \cdot \frac{\nabla P}{\rho} \boldsymbol{b}_{j} \cdot\left(\frac{\nabla P}{\Gamma P}-\frac{\nabla \rho}{\rho}\right)=0$.

We have now assembled all the quantities needed to calculate the coefficients $\left(C_{0}, E_{i, j}, i=1,2, j=1,2\right)$ contained in the linear governing Eqs. (51) and (52) for $\alpha$ and $\beta$. These can be constructed using Eqs. (46)-(54) of Sect. 4. We obtain:

$$
\begin{aligned}
E_{11}= & -\frac{G M_{*}}{r^{3}} \frac{\left(1+2 e \cos \varphi+e^{2}\left(1-3 \sin ^{2} \varphi\right)\right)}{1+e^{2}+2 e \cos \varphi} \\
& +\frac{G M_{*}}{r^{3}} \frac{(1+e \cos \varphi)^{3}}{\left(1+e^{2}+2 e \cos \varphi\right)^{2}}, \\
E_{12}= & \frac{G M_{*}}{r^{3}} \frac{3 K_{z} e \sin \varphi(1+e \cos \varphi)}{N\left(1+e^{2}+2 e \cos \varphi\right)}+\dot{\boldsymbol{b}}_{1} \cdot \dot{\boldsymbol{b}}_{2}-\frac{D C_{0}}{D t}, \\
E_{21}= & \frac{G M_{*}}{r^{3}} \frac{3 K_{z} e \sin \varphi(1+e \cos \varphi)}{N\left(1+e^{2}+2 e \cos \varphi\right)}+\dot{\boldsymbol{b}}_{1} \cdot \dot{\boldsymbol{b}}_{2}+\frac{D C_{0}}{D t}
\end{aligned}
$$

and

$$
E_{22}=\frac{G M_{*}}{r^{3}} \frac{K_{z}^{2}\left(2+4 e \cos \varphi+e^{2}\left(2-3 \sin ^{2} \varphi\right)\right)}{N^{2}\left(1+e^{2}+2 e \cos \varphi\right)}-\boldsymbol{b}_{2} \ddot{\boldsymbol{b}}_{2} .
$$

\section{Appendix B: Parametric instability for nearly circular streamlines}

We here consider the generic parametric instability that occurs for streamlines near to circles for systems with inertia/gravity modes. But note that as indicated for the specific example of Keplerian ellipses instability may persist for significant departures of the streamlines from circles.

We start with the governing equations in vector form (see Eq. (77) in Sect. 7). These are written as:

$\ddot{\boldsymbol{y}}-2 C_{0} \boldsymbol{e}_{3} \times \dot{\boldsymbol{y}}=\boldsymbol{E} \cdot \boldsymbol{y}$

where the two dimensional vector $\boldsymbol{y}^{T}=(\alpha, \beta)$ and the vector $\boldsymbol{e}_{3} \times \dot{\boldsymbol{y}}=(-\dot{\beta}, \dot{\alpha})^{T}$. The two dimensional matrix $\boldsymbol{E}$ has elements $E_{11}, E_{12}, E_{21}, E_{22}$ as defined in Sect. 4.4 above.

For circular streamlines $\boldsymbol{E}=(\boldsymbol{E})_{c}$ and $C_{0}=C_{0, c}$ are constant, while for small departures from circularity we can expand to first order in some small parameter $\epsilon$. When the streamlines are Keplerian ellipses $\epsilon \equiv e$. We thus write $C_{0}=$ $C_{0, c}+\epsilon C_{0,1}$ and $\boldsymbol{E}=\boldsymbol{E}_{c}+\epsilon \mathcal{E}$. Here $C_{0,1}$ and $\mathcal{E}$ are periodic functions of time where the period is just the time it takes a fluid element to orbit around a streamline. Without loss of generality we may assume that the time average of these coefficients over one period is zero. For the Keplerian ellipse streamlines, from the results given in Appendix A, $C_{0, c}=\Omega K_{z} / K$, and the only non zero element of $(\boldsymbol{E})_{c}$ is $\left(E_{22}\right)_{c}=3\left(\Omega K_{z} / K\right)^{2}$. Here $\Omega=\sqrt{G M_{*} / a^{3}}$ where $a$ is the semi major axis of the streamline of interest. Similarly, making the equivalence $\epsilon \equiv e$, $C_{0,1}=\Omega\left(K_{z} / K\right)^{3} \cos \varphi$ while the elements of $\mathcal{E}$ are given by $\mathcal{E}_{11}=-\Omega^{2} \cos \varphi, \mathcal{E}_{12}=\Omega^{2}\left(K_{z} / K^{3}\right) \sin \varphi\left(4 K_{z}^{2}+2 K_{r}^{2}\right), \mathcal{E}_{21}=$ $2 \Omega^{2}\left(K_{z} / K\right) \sin \varphi$ and $\mathcal{E}_{22}=2 \Omega^{2}\left(K_{z}^{2} / K^{4}\right) \cos \varphi\left(4 K_{z}^{2}+K_{r}^{2}\right)$.

Note that we may write to this order $\varphi=\Omega t$ making the coefficients that are proportional to the eccentricity vary harmonically with time. The period is simply the orbital period on the streamline. We now write the governing equations in the form

$\ddot{\boldsymbol{y}}-2 C_{0, c} \boldsymbol{e}_{3} \times \dot{\boldsymbol{y}}-(\boldsymbol{E})_{c} \cdot \boldsymbol{y}=2 \epsilon C_{0,1} \boldsymbol{e}_{3} \times \dot{\boldsymbol{y}}+\epsilon \mathcal{E} \cdot \boldsymbol{y}$

and treat the right hand side using perturbation theory.

We first remark that when $\epsilon=0$, there is a simple normal mode solution appropriate to the time independent case which may be written in the form

$\boldsymbol{y}=\boldsymbol{y}_{0} \exp (i \sigma t)$

where $\boldsymbol{y}_{0}$ is a constant vector and $\sigma$ is a normal mode frequency which satisfies

$-\sigma^{2} \boldsymbol{y}_{0}-2 i \sigma C_{0, c} \boldsymbol{e}_{3} \times \boldsymbol{y}_{0}-(\boldsymbol{E})_{c} \cdot \boldsymbol{y}_{0}=0$.

In line with the example presented in Sect. 5, we expect this mode to correspond to an inertial/gravity mode. The spectrum is dense and so we may always expect to be able to choose $\sigma$ to be close to $\Omega / 2$ which is the lowest order commensurability condition for parametric resonance (for the example of section 5 the commensurability is exact for $K_{r} / K_{z}=\sqrt{3}$ ).

We now move on to consider the case of $\epsilon$ non zero but small and set $\sigma=\Omega / 2+\delta \sigma$, where $\delta \sigma=O(\epsilon \Omega)$. 
We now seek a solution of Eq. (B.2) in the form

$\boldsymbol{y}=A(t) \boldsymbol{y}_{0} \exp (i \Omega t / 2)+c c .+\sum_{n=1}^{\infty} \epsilon^{n} \boldsymbol{y}_{n}$.

Here $A(t)$ is a slowly varying amplitude function to be determined and here and below $c c$ denotes the addition of the complex conjugate of the first expression ensuring that as required $y$ is real. The terms $\epsilon^{n} \boldsymbol{y}_{n}$, assumed real are additions that are of first and higher order in $\epsilon$.

By stating that $A$ is slowly varying we mean that $|\dot{A} / A|=$ $O(\epsilon \Omega)$ and $|\ddot{A} / A|=O(\epsilon \Omega)^{2}$.

Given this we substitute Eqs. (B.5) into (B.2) and drop expressions that are clearly second or higher order in $\epsilon$ to get an equation for $A$. This reads

$$
\begin{array}{r}
\left(i \Omega \boldsymbol{y}_{0}-2 C_{0, c} \boldsymbol{e}_{3} \times \boldsymbol{y}_{0}\right)(\dot{A}-i \delta \sigma A) \exp (i \Omega t / 2)+c c \\
=\epsilon\left(i \Omega C_{0,1} \boldsymbol{e}_{3} \times \boldsymbol{y}_{0}+\mathcal{E} \boldsymbol{y}_{0}\right) A \exp (i \Omega t / 2)+c c \\
-\epsilon\left(\ddot{\boldsymbol{y}}_{1}-2 C_{0, c} \boldsymbol{e}_{3} \times \dot{\boldsymbol{y}}_{1}-(\boldsymbol{E})_{c} \cdot \boldsymbol{y}_{1}\right) .
\end{array}
$$

We now multiply by $) \exp (-i \Omega t / 2)$ and take a time average over many orbital periods, assuming $A$ varies slowly enough that it can be factored out, to obtain

$$
\begin{aligned}
& \left(i \Omega \boldsymbol{y}_{0}-2 C_{0, c} \boldsymbol{e}_{3} \times \boldsymbol{y}_{0}\right)(\dot{A}-i \delta \sigma A)= \\
& \quad\left\langle\epsilon\left(-i \Omega C_{0,1} \boldsymbol{e}_{3} \times \boldsymbol{y}_{0}^{*}+\mathcal{E} \boldsymbol{y}_{0}^{*}\right) \exp (-i \Omega t)\right\rangle A^{*} \\
& \quad-\epsilon\left\langle\left(\ddot{\boldsymbol{y}}_{1}-2 C_{0, c} \boldsymbol{e}_{3} \times \dot{\boldsymbol{y}}_{1}-(\boldsymbol{E})_{c} \cdot \boldsymbol{y}_{1}\right) \exp (-i \Omega t / 2)\right\rangle
\end{aligned}
$$

where the angular brackets denote the time average over many orbital periods. Because $C_{0,1}$ and $\mathcal{E}$ vary harmonically with angular frequency $\Omega$, it is anticipated that the time averages retained above are not zero.

Setting $A=A_{0} \exp (\lambda t)$ in Eq. (B.7), $\lambda$ being the growth rate, taking the scalar product with $\boldsymbol{y}_{0}^{*}$, which as can be shown by doing the time integration by parts reduces the final term involving $\boldsymbol{y}_{1}$ to be $O\left(\epsilon^{2}\right)$ and finally taking the modulus of the result gives an expression for $\lambda^{2}$ correct to $O\left(\epsilon^{2}\right)$ in the form

$$
\lambda^{2}=\frac{\left|\left\langle\boldsymbol{\epsilon} \boldsymbol{y}_{0}^{*} \cdot \mathcal{E} \boldsymbol{y}_{0}^{*} \exp (-i \Omega t)\right\rangle\right|^{2}}{\left.|i \Omega| \boldsymbol{y}_{0}\right|^{2}-2 C_{0, c} \boldsymbol{y}_{0}^{*} \cdot \boldsymbol{e}_{3} \times\left.\boldsymbol{y}_{0}\right|^{2}}-\left(\delta \sigma^{2}\right) \text {. }
$$

Thus parametric instability occurs ( $\lambda$ is real) at a maximum rate when the commensurability is exact or $\sigma=\Omega / 2$ and for fixed $\epsilon$ the frequency half-width associated with instability corresponds to this maximum growth rate in magnitude. The growth rate itself is $\propto \epsilon$.

It is easy to evaluate Eq. (B.8) for the Keplerian streamline model of Sect. 6 . We begin by noting that $\boldsymbol{y}_{0}$ can be found from the solution for the local oscillation mode given for the circular streamline case in Sect. 5 (noting that $\Omega^{\prime}=-3 \Omega /(2 r)$ for the Keplerian case). From that $\boldsymbol{y}_{0}^{T}=\left(2 i K_{z} \Omega, K \sigma\right)$.

It is then straightforward to evaluate $\lambda$ given $\boldsymbol{y}, C_{0, c}$ and $\mathcal{E}$ as specified above. One obtains

$\lambda^{2}=9 e^{2} \Omega^{2} / 256-\left(\delta \sigma^{2}\right)$,

giving a peak growth rate as a function of eccentricity given by $\lambda_{\max }=3 e \Omega / 16$. The instability band width is then given by $\delta \sigma=\lambda_{\max }$.

\section{Appendix C: Relation to the spectrum of the global problem}

We have studied the stability of fluid displacements that are localized around the vicinity of fluid streamlines and circulate around with the fluid. When conditions vary periodically around streamlines, parametric excitation becomes a possibility aided by an underlying mode spectrum that is dense so that resonance conditions may always be satisfied. The analysis was done in a local limit of high wavenumber in directions orthogonal to streamlines. However, it is possible to relate the local spectrum so found to that of the global problem. A physical reason for this is that it is possible to take a local limit where wavenumbers tend to infinity but mode oscillation frequencies remain finite. This naturally results in a limit in which the group velocity vanishes and hence localization becomes possible. We here illustrate the connection between the local and global problems.

The equation satisfied by the Lagrangian displacement $\boldsymbol{\xi}$ is Eq. (18) which after use of Eq. (19) and replacement of the convective derivatives through Eqs. (22) and (23) yields an operator equation of the form

$\mathcal{L}(\boldsymbol{\xi}, \sigma)=0$,

where we have included the $\sigma$ dependence to emphasize that we have an eigenvalue problem for $\sigma$ and that the determined eigenvalues constitute the spectrum of the system. We adopt the inner product defined through Eq. (25) to define the norm $|\boldsymbol{\xi}|=|(\boldsymbol{\xi}, \boldsymbol{\xi})|$.

Then following Papaloizou \& Pringle (1982) and Terquem \& Papaloizou (1996), we remark that the spectrum coincides with the values of $\sigma$ for which the operator $\mathcal{L}$ has an unbounded inverse. Further if one can find any $\boldsymbol{\xi}$ for which $|\mathcal{L}(\boldsymbol{\xi}, \sigma)|^{2} /|\boldsymbol{\xi}|^{2}$ can be made as small as we please then $\mathcal{L}$ will have an unbounded inverse and so $\sigma$ belongs to the spectrum.

In fact displacements of the form (26) which are localized on streamlines over a length scale that tends to zero as a fractional power of the wave number parameter $\lambda^{-1}$, which itself scales as the inverse wavenumber as that approaches zero, can be used to construct a set of normalized $\boldsymbol{\xi}$ which lead to $|\mathcal{L}(\xi, \sigma)|^{2} \rightarrow 0$ as $\lambda^{-1} \rightarrow 0$ (see Papaloizou \& Pringle 1982; Terquem \& Papaloizou 1996, for other examples).

In this limit $\boldsymbol{\xi}$ is of the form (39) and is obtained from the eigenvalue problem (79), as is $\sigma$, which then belongs to the spectrum of $\mathcal{L}$.

\section{References}

Artymowicz, P. 1992, PASP, 104, 769

Balbus, S. A., \& Hawley, J. F. 1991, ApJ, 376, 214

Fromang, S., Terquem, C., \& Balbus, S. A. 2002, MNRAS, 329, 18

Gammie, C. F. 1996, ApJ, 462, 725

Goldreich, P., \& Tremaine, S. 1980, ApJ, 241, 425

Goldreich, P., \& Sari, R. 2003, ApJ, 585, 1024

Goodman, J. 1993, ApJ, 406, 596

Gurzadyan, V. G., \& Ozernoy L. M. 1979, Nature, 280, 214

Hawley, J. F. 2000, ApJ, 528, 462 
Kumar, P., \& Goodman, J. 1996, ApJ, 466, 946

Lin, D. N. C., \& Papaloizou, J. C. B. 1993, Protostars and Planets III, 749 (Tucson, AZ: University of Arizona Press)

Lin, D. N. C., Papaloizou, J. C. B., \& Kley, W. 1993, ApJ, 689

Lynden-Bell, D., \& Ostriker, J. P. 1967, MNRAS, 136, 293

Marcy, G. W., \& Butler, R. P. 1998, ARA\&A, 36, 57

Marcy, G. W., \& Butler, R. P. 2000, PASP, 112, 137

Mayor, M., \& Queloz, D. 1995, Nature, 378, 355

Ogilvie, G. I. 2001, MNRAS, 325, 2310

Okazaki, A. T. 1991, PASJ, 43, 750

Papaloizou, J. C. B. 1973, MNRAS, 162, 143P

Papaloizou, J. C. B. 2002, A\&A, 388, 615

Papaloizou, J. C. B. 2005, A\&A, 432, 757

Papaloizou, J. C. B., Nelson, R. P., \& Masset, F. S. 2001, A\&A, 366, 263
Papaloizou, J., \& Pringle, J. E. 1980, MNRAS, 196, 371P

Papaloizou, J., \& Pringle, J. E. 1982, MNRAS, 100, 49

Papaloizou, J. C. B., Savonije, G. J., \& Henrichs, H. F. 1992, A\&A, 265, L45

Ryu, D., \& Goodman, J. 1994, ApJ, 422, 269

Steinacker, A., \& Papaloizou, J. C. B. 2002, ApJ, 571, 413

Tassoul, J. L. 1978, Theory of Rotating Stars (Princeton: Princeton Univ, Press)

Terquem, C., \& Papaloizou, J. C. B. 1996, MNRAS, 279, 767

Ward, W. R. 1997, Icarus, 126, 261

Whitehurst, R. 1988, MNRAS, 232, 35

Whitaker, E. T., \& Watson, G. N. 1996, A Course of Modern Analysis (Cambridge: Cambridge University Press)

Wu, Y., \& Goldreich, P. 2001, ApJ, 546, 469

Vandakurov, Y. V. 1967, ApJ, 149, 435 